\title{
Occupational therapy: The process in acute psychiatry
}

\author{
Z Syed, BSc OT, MOT, PG Dip Addiction Care \\ Division of Occupational Therapy, Department of Health and Rehabilitation Sciences, Faculty of Health Sciences, University of Cape Town, South Africa
}

Corresponding author: Z Syed (zari.syed@uct.ac.za)

\begin{abstract}
Background. The role of the occupational therapist in the acute psychiatric setting includes individual and group assessment and intervention, discharge planning and community reintegration. However, there is a lack of understanding around specific types of assessment and interventions commonly used in this setting, as well as a lack of research into students' understanding of the occupational therapy process in the acute psychiatric context.

Objective. To explore undergraduate occupational therapy students' experiences of the implementation of the occupational therapy process in the acute psychiatric setting.

Methods. A qualitative approach was used. The setting included two acute psychiatric wards in Western Cape Province, South Africa. Data sources were: a focus group, semi-structured interviews with each of the 4 participants and students' weekly reflective journals. Thematic analysis of all 3 data sources was employed.

Results. Students identified the institutional barrier of rapid discharge of clients as one of the main barriers influencing the implementation of the occupational therapy process. Discharge from the acute psychiatric wards occurred before comprehensive intervention, which influenced how they understood implementation of the occupational therapy process. Four subthemes were identified: assessment, goal setting, intervention and discharge. Conclusion. Priority assessment methods and interventions were identified that are seen as unique to the occupational therapy profession. This study contributes towards the body of evidence in understanding students' experiences of implementing the occupational therapy process in the acute psychiatric setting.
\end{abstract}

Afr J Health Professions Educ 2020;12(1):9-11. https://doi.org/10.7196/AJHPE.2020.v12i1.1185

The role of the occupational therapist in the acute psychiatric setting includes individual and group assessment and intervention, discharge planning and community reintegration. ${ }^{[1]}$ However, there is a lack of understanding around specific types of assessment and interventions commonly used in this setting, where the occupational therapist does not have the opportunity to predict a predetermined programme that mirrors the traditional occupational therapy process. ${ }^{[2]}$ The occupational therapist and the students should have flexibility in practice. ${ }^{[3]}$ There is a lack of research into the students' understanding of the occupational therapy process in the acute psychiatric context.

\section{Methods}

This study explored third-year occupational therapy students' experiences in the acute psychiatric setting. A qualitative approach was used, which allowed participants to share their subjective perspectives. ${ }^{[4]}$ The setting comprised two acute admission wards at a tertiary psychiatric hospital in Western Cape Province, South Africa. The study population was a thirdyear occupational therapy class at an institute for higher learning in the Western Cape. Participants were selected using purposive sampling, based on their placement in the acute psychiatric fieldwork setting. A sample size of 4 viable participants was identified. ${ }^{[5]}$

\section{Data collection}

Focus group discussions and semi-structured interviews with each of the 4 participants were used for data gathering. Students' electronic-format reflective journals were collected as a third form of data. Students were asked about the enablers and limitations with the implementation of the occupational therapy process in the setting.

\section{Data analysis}

The 4 interviews and focus group discussions were digitally recorded and transcribed verbatim. Data were analysed using inductive, thematic analysis, which complemented the research study ${ }^{[4]}$ Electronic journals were used by the researcher to collate information gained from the semistructured interviews and focus group discussions. Trustworthiness was ensured using the principles of transferability, credibility and confirmability. ${ }^{[4]}$ Purposive sampling ensured transferability. Data triangulation illustrated the application of credibility. Conformability was guaranteed by means of an audit trail. Reflexivity was considered because of the role of the researcher as a fieldwork supervisor at the same institution where the participants were completing their course. ${ }^{[4]}$ The researcher was not allocated the role of fieldwork supervisor of the participants during this period.

\section{Ethical approval}

Ethical approval was obtained from Stellenbosch University's Health Research Ethics Committee (ref. no. S14/03/059). Informed consent was obtained from the participants. ${ }^{[6]}$

\section{Results}

Students identified the phenomenon of rapid discharge of clients as one of the main institutional obstacles affecting the implementation of the occupational therapy process. Furthermore, students reported that clients 
seemed to be discharged before comprehensive intervention, which initially hindered how they understood the implementation of the occupational therapy process. Four subthemes were identified: assessment, goal setting, intervention and discharge.

\section{Assessment}

Students reported that the high turnover rate resulted in restrictive time frames, where they needed to adapt their process to maximise engagement with clients. The challenge identified by students in relation to the restricted time frames appeared to modify their skills of assessing clients in a short period of time. Therefore, students reasoned that the use of group sessions was a means of adapted assessment that was efficient to observe the behaviours of clients. Adapted, individual assessments were also carried out with methods such as functional observation, interviews and activity clocks:

'I had to adapt the assessment, and chose to discuss the relevant issue.' (Peter, journal 2)

\section{Goal setting}

The institutional barrier of a high turnover of clients appeared to be difficult for students to manage when learning about the importance of specific, measurable, appropriate, realistic and time-sensitive (SMART) goal setting as part of the occupational therapy process. Students further reported having to be creative and think very concretely when planning goals. They also observed that, because of the short-term nature of the acute setting, clients' discharge was fast tracked. Consequently, the practice of goal-setting skills seemed to be difficult to implement:

'Psychiatry, the biggest difficulty I've had at the moment is having to write up goals on a client and then going the next day and finding out the client has been discharged.' (David, interview)

\section{Intervention}

Students identified interventions, including reality orientation and psychoeducation, which were found to be most appropriate to address deficits. Providing reality-orientation intervention to clients who seemed to be experiencing psychotic symptoms tends to be considered a part of occupational therapy. Psychoeducation is one of the occupational therapy interventions that appeared to be provided to clients in the setting. Students reported that psychoeducation included educating clients about their conditions, and the impact on their occupational functioning was seen to be instrumental in maintaining clients' health. Accordingly, students highlighted that the improvement of clients' functional performance in activities such as self-care was a focus of intervention in relation to occupation:

'It's [intervention] very much focused on functional performance and how we can put them back into their premorbid condition.' (Julia, interview)

\section{Discharge}

Acceleration of clients' discharge a few days after admission was perceived as one of the institutional barriers identified by students. The high pace of the ward process led to the therapeutic process being more fast tracked than anticipated. This seemed to make the students feel frustrated by the reality of the healthcare system:

'The frustration of high turnover is quite a challenging experience of the acute psychiatry block.' (David, interview)

\section{Discussion}

A short admission period appears to be a global phenomenon. ${ }^{[2]}$ Accordingly, occupational therapists and students need to evolve to meet the demands of such strains on mental healthcare.

The institutional barrier of the high turnover in the wards was one of the key aspects that influenced students' learning regarding the role of occupational therapy in this setting. The initial stage of assessment appeared to be restricted owing to the uncertain, impending daily discharge of clients. Institutional expectations regarding the types of assessment techniques students could practise were discussed. The literature indicated that the use of formal assessment tools in this setting tends to be tricky. ${ }^{[2]}$ Students acknowledged that the use of standardised assessments was not the norm. Assessment of function is the main priority in the short space of time assigned. ${ }^{[2]}$ Students reported that they adapted assessment methods and made use of more functional, activity-based assessments to gain information regarding the clients' occupational performance.

Being aware of the limited time frames during which clients were in the setting, allowed students to consider goals that had a measure to determine the degree of achievement. The involvement of clients in the therapeutic process suggests a client-centred approach. ${ }^{[7]}$ Students indicated that within the limited time frames, goals needed to be set with clients to identify priority occupation needs where functional deficit was evident. Providing clients with a forum in which to engage about their goals creates the opportunity for them to analyse their strengths and limitations. This leads to more goal-directed activities during the intervention phase..$^{[7]}$

Students highlighted addressing occupational functional deficit as a focus in their quest to understand intervention in this setting. Reality orientation, psychoeducation and facilitation of functional self-care were identified as important interventions. ${ }^{[2,8]}$ Students reported that intervention with clients in areas of occupation such as self-care was a primary role of the occupational therapist. They also have the scope to enable a client to engage in functional self-care that is essential to improve performance. ${ }^{[2]}$ Psychoeducation is stressed in the literature as an effective intervention in this setting. One of the roles of the occupational therapist is to facilitate clients' understanding of their illness. ${ }^{[8]}$ Students reported that educating clients regarding their diagnosis was significant in improving health maintenance as an occupation. Reality orientation appeared to be an enabler that facilitated clients having continuous awareness of person, place and time, which are important in the management of psychosis. ${ }^{[2]}$ Therefore, the students felt that reality orientation was another integral part of intervention with clients that was unique to occupational therapy, which led to the improvement of deficits in other areas of occupation.

Discharge planning was highlighted as a priority in the occupational therapy process in the acute psychiatric setting. ${ }^{[1]}$ Students were able to consider the community occupational therapist as a valuable resource in the intervention process regarding clients. This may mean that there is a need for collaboration between those in the acute psychiatric setting and community occupational therapists. ${ }^{[1]}$

\section{Conclusion}

The occupational therapy process in an acute psychiatric setting was delineated to assessment, goal setting, intervention and discharge planning. Priority assessment methods and interventions were identified that are seen as unique to the occupational therapy profession. It is recommended that 


\section{Short Research Report}

mental health coursework should include a stronger focus on the role of the occupational therapist in the acute psychiatric context. Use of the identified and adapted assessment and intervention techniques is also suggested. Further research with regard to student experiences in acute psychiatric settings and their experience of adapting to institutional barriers would enhance student experiences of service delivery.

Declaration. This article is based on a study done by ZS in partial fulfilment of her Master's degree in occupational therapy.

Acknowledgements. The author would like to acknowledge the participating students for their contribution. Thanks is extended to the Department of Occupational Therapy at the institute of higher learning where the study was conducted for their permission to work with the students.

Author contributions. Mrs Nicola Vermeulen and Ms Juanita Bester assisted in supervising the author during the research study from which this article originated.

\section{Funding. None.}

Conflicts of interest. None.

1. Lloyd C, Williams PL. Occupational therapy in the modern adult acute mental health setting: A review of curren practice. Int J Ther Rehabil 2010;17(9):483-493. https://doi.org/10.12968/iitr.2010.17.9.78038

Shorten C, Crouch R. Acute psychiatry and the dynamic short term intervention of the occupational therapist. In Crouch R, Alers V. Occupational Therapy in Psychiatry and Mental Health. NJ, USA: John Wiley, 2014:115-123. 3. Bryant W, Cordingley $\mathrm{K}$, Sims $\mathrm{K}$, et al. Collaborative research exploring mental health service use perspectives on acute inpatient occupational therapy. Br J Occ Ther 2016;79(10):607-613. https://dol. org/10.1177/0308022616650899

4. Creswell JW. Designing a qualitative study. In: Creswell JW. Qualitative Inquiry and Research Design: Choosing Among Five Approaches. Thousand Oaks, CA: Sage, 2006:42-68.

5. Sandelowski M. Sample size in qualitative research. Res Nurs Health 1995;18(2):179-183. https://doi.org/10.1002/ nur.4770180211

6. World Medical Association. World Medical Association Declaration of Helsinki: Ethical principles for medical research involving human subjects. JAMA 2013;310(20):2191-2194. https://doi.org/10.1001/jama.2013.281053 7. Robinson AM, Avallone J. Occupational therapy in acute inpatient psychiatry: An activities health approach Am J Occ Ther 1990;44(9):809-814. https://doi.org/10.5014/ajot.44.9.809

8. Eaton P. Psychoeducation in acute mental health settings: Is there a role for occupational therapists? $\mathrm{Br} J \mathrm{Occ}$ The 2002;65(7):321-326. https://doi.org/10.1177/030802260206500704

Accepted 4 September 2019. 